\section{COMORBILIDAD DE TUBERCULOSIS Y DIABETES EN UN HOSPITAL DE COLOMBIA}

\author{
TUBERCULOSIS AND DIABETES \\ COMORBIDITY IN A HOSPITAL OF COLOMBIA
}

\author{
Dayron Fernando Martínez-Pulgarín ${ }^{1, a}$, \\ John Alejandro Rico-Gallego, ${ }^{1, a}$, \\ Felipe Vera-Polanía ${ }^{1, a}$, Javier Alejandro Gaviria ${ }^{2, b}$, \\ Alfonso J. Rodríguez-Morales ${ }^{1, c}$
}

Sr. Editor. Hemos leído con interés el artículo de Ugarte-Gil y Moore (1) que discute la importancia de la comorbilidad diabetes mellitus/tuberculosis (DM/TB). Es por ello que quisiéramos continuar su discusión haciendo algunas consideraciones al respecto, especialmente desde la perspectiva de la falta de investigación de otras comorbilidades infecciosas, en adición a la TB, pero además mostrando cuál es la situación de dicha comorbilidad en un hospital de Colombia.

La DM incrementa la susceptibilidad a diversas infecciones. Por ello, el aumento en su prevalencia puede ser importante en el riesgo de comorbilidades infecciosas, como la TB. La hiperglicemia y la insulinopenia celular, así como los efectos indirectos en la afectación de la quimiotaxis, fagocitosis, activación y presentación de antígenos, de linfocitos y macrófagos, predisponen a la TB ${ }^{(1)}$, pero también a infecciones como la piomiositis (2) e incluso parasitosis como la estrongiloidiasis y la malaria ${ }^{(3,4)}$.

La infección por el virus de inmunodeficiencia humana $(\mathrm{VIH})$ cumple un papel importante en la reemergencia de la TB, por lo cual, en el contexto de la evaluación de DM/ TB, ella también tiene implicaciones. Se debe reflexionar sobre las consecuencias de dichas comorbilidades ${ }^{(1)}$. Se requieren llevar a cabo investigaciones que, en primera instancia, permitan conocer la magnitud epidemiológica y clínica del problema.

Realizamos una revisión del registro de pacientes con diagnóstico de DM del Hospital Santa Mónica, principal

\footnotetext{
1 Semillero de Investigación SIDA y Otras Enfermedades Infecciosas, Facultad de Ciencias de la Salud, Universidad Tecnológica de Pereira. Risaralda, Colombia.

2 E.S.E. Hospital Santa Mónica. Risaralda, Colombia.

a Estudiante de Medicina; ${ }^{\text {b }}$ odontólogo; ${ }^{\mathrm{c}}$ médico tropicalista Recibido: 09-04-14 Aprobado: 23-04-14
}

Citar como: Martínez-Pulgarín DF, Rico-Gallego JA, Vera-Polanía F Gaviria JA, Rodríguez-Morales AJ. Comorbilidad de tuberculosis y diabetes en un hospital de Colombia [carta]. Rev Peru Med Exp Salud Publica. 2014;31(2):397-8. centro hospitalario de Dosquebradas, segunda ciudadmunicipio del departamento de Risaralda, Colombia; se revisaron los códigos de la clasificación internacional de enfermedades (CIE-10), correspondientes (A15-A19, B20, B90, J65, P37 y Z03), con el fin de determinar cuál había sido la prevalencia de TB (cualquier forma) en los pacientes con DM. Del año 2008 a 2012 se diagnosticaron 43379 pacientes con DM, de los cuales 183 tuvieron TB $(0,4 \%$ o 42 casos $/ 10000$ pacientes), diagnosticándose, en promedio, 37 casos de TB en pacientes con DM por año (mínimo 26, máximo 45). De los casos con comorbilidad, 92,3\% $(169,39$ casos $/ 10000$ pacientes) de ellos eran formas pulmonares confirmadas por microscopía (A15.0); 2,2\% (4) sin diagnóstico bacteriológico/histológico (A16.0, A16.2); seguidos por casos ganglionares (A15.4) $(1,1 \%)$ (2), meningitis tuberculosa (A17.0) $(1,1 \%)(2)$, TB en otros órganos (A18.8) (1,1\%) (2), TB cutánea (A18.4) $(0,55 \%)(1)$ (en total siete extrapulmonares), un caso de TB en un paciente con DM e infección por VIH/SIDA (B20.0) $(0,55 \%)$, y dos casos con sospecha clínica de TB (Z 03.0 y Z 11.1) (1,1\%).

Estudios recientes en la India han encontrado que la comorbilidad pueden alcanzar cifras tan altas como $29 \%$ (5). Dosquebradas no es un municipio con alta carga de TB (21,1 casos/100 000 hab, 2009), por lo cual será importante hacer estudios similares, en otros lugares donde se presentan altas tasas de incidencia de TB (ej. La Virginia, 50,7 casos/100 000 hab, 2009). El artículo de Ugarte y Moore ${ }^{(1)}$, nos estimuló a iniciar investigaciones que puedan establecer la importancia epidemiológica de dicha comorbilidad en Colombia, que permitirá intervenir en la reducción de su impacto en nuestras poblaciones.

Fuentes de financiamiento: Universidad Tecnológica de Pereira, Pereira, Risaralda, Colombia y ESE Hospital Santa Mónica, Dosquebradas, Risaralda, Colombia.

Conflictos de interés: los autores declaran no tener conflictos de interés con la publicación de este artículo.

\section{REFERENCIAS BIBLIOGRÁFICAS}

1. Ugarte-Gil C, Moore DA. Comorbilidad de tuberculosis y diabetes: problema aún sin resolver. Rev Peru Med Exp Salud Publica. 2014;31(1):137-42.

2. Polizelli DV, Geraldino GC, Narvaes E, Funes E, de Toledo RA, Menin R. Pyomyositis associated with diabetes mellitus and liver cirrhosis. Bras J Rheumatol. 2010;50(4):475-7.

3. Mendonça SC, Gonçalves-Pires Mdo R, Rodrigues RM, Ferreira A Jr, Costa-Cruz JM. Is there an association between positive Strongyloides stercoralis serology and diabetes mellitus? Acta Trop 2006; 99(1):102-5. 
4. Danquah I, Bedu-Addo G, Mockenhaupt FP. Type 2 diabetes mellitus and increased risk for malaria infection. Emerg Infect Dis. 2010 Oct;16(10):1601-4. doi: 10.3201/eid1610.100399.

5. Raghuraman S, Vasudevan KP, Govindarajan S, Chinnakali P, Panigrahi KC. Prevalence of Diabetes Mellitus among Tuberculosis Patients in Urban Puducherry. N Am J Med Sci. 2014 6(1):30-4.

Correspondencia: Alfonso J. Rodriguez-Morales

Dirección: Departamento de Medicina Comunitaria, Piso 3, Facultad de Ciencias de la Salud, Universidad Tecnológica de Pereira, Pereira, Risaralda, Colombia.

Teléfono: 573008847448

Correo electrónico: ajrodriguezmmd@gmail.com

\section{LA SALUD AMBIENTAL EN EL PERÚ}

\section{ENVIRONMENTAL HEALTH IN PERU}

\author{
Gustavo F. Gonzales ${ }^{1,2,3, a}$, Kyle Steenland ${ }^{4, b}$
}

Sr. Editor. Del 28 al 30 de abril de 2014 se llevó a cabo en Lima una reunión de expertos para presentar las investigaciones que se desarrollan en Perú sobre contaminación del aire, contaminación del agua y el impacto del cambio climático/variabilidad climática y su impacto en salud y las prioridades de investigación que se pueden establecer en la actualidad. En este taller participaron investigadores y funcionarios de costa, sierra, selva y del norte, centro y sur del país. Las exposiciones pueden ser visualizadas en http:// geohealthperu.wordpress.com /exposiciones-del-taller/ taller-de-aire/.

Uno de los problemas que se resaltó fue la alta contaminación de Lima, pues los valores de material particulado (PM 2,5) estaban por encima de la norma tanto del Perú $\left(20 \mathrm{ug} / \mathrm{m}^{3}\right)$ como de la OMS $\left(10 \mathrm{ug} / \mathrm{m}^{3}\right)$. Esto ha salido a relucir con el informe de OMS ${ }^{(1)}$ que indica que Lima tiene la mayor contaminación del aire exterior por el PM 2,5 (materia particulada mayor de 2,5 micrones)

\footnotetext{
1 Instituto de Investigaciones de la Altura. Lima, Perú.

2 Facultad de Ciencias y Filosofía, Universidad Peruana Cayetano Heredia. Lima, Perú.

Academia Nacional de Ciencias. Lima, Perú.

4 Rollins School of Public Health, University of Emory. EE. UU.

a Doctor en Ciencias y doctor en Medicina; ${ }^{\mathrm{b}} \mathrm{PhD}$

Recibido: 12-05-14 Aprobado: 28-05-14
}

Citar como: Gonzales GF, Steenland K. La salud ambiental en el Perú [carta]. Rev Peru Med Exp Salud Publica. 2014;31(2):398-9.

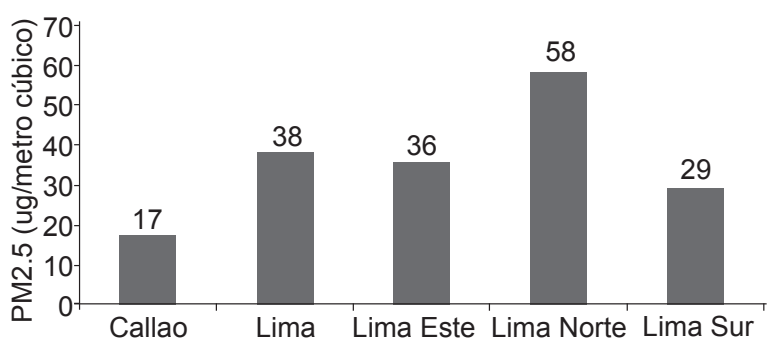

Figura 1. Niveles de PM 2,5 en Lima y Callao. Fuente: OMS (1)

de América Latina $\left(38 \mathrm{ug} / \mathrm{m}^{3}\right)$. El mismo reporte muestra que la zona de Lima Norte es la más contaminada de Lima (Figura 1). La medición de las partículas finas de 2,5 micrómetros o menos de diámetro (PM 2,5) es considerado como el mejor indicador del nivel de riesgos para la salud derivados de la contaminación del aire.

Es de notar que en abril de 2014, la OMS publicó un informe donde se estima que la contaminación del aire exterior era responsable de la muerte de unos 3,7 millones de personas menores de 60 años a nivel global ${ }^{(2)}$. Dado los hallazgos de la pendiente exposición-respuesta (PM 2,5-muerte) de otros países ${ }^{(3)}$, y el nivel promedio de PM 2,5 en el aire de Lima en los últimos 10 años, en exceso a la norma de OMS (50 ug $/ \mathrm{m}^{3}$ frente a $\left.10 \mathrm{ug} / \mathrm{m}^{3}\right)$, estimamos que el riesgo relativo de los habitantes de Lima para la muerte cardiopulmonar sería 1,25. Usando la fórmula para calcular la fracción atribuible ${ }^{(4)}$, hemos calculado que en Lima el nivel excesivo de PM 2,5 resulta en aproximadamente 2300 muertes prematuras anuales.

Además, entre las personas que usan combustible de biomasa en la cocina, como leña, bosta, o champa, que representa aproximadamente 10 millones de personas ${ }^{(5)}$, estimamos que la exposición a PM 2,5 dentro de las casas (promedio $100 \mathrm{ug} / \mathrm{m}^{3}$ ) resulta en aproximadamente 3000 muertes anuales prematuras entre adultos, sin contar otro numero no conocido de muertes entre niños por enfermedades respiratorias, principalmente neumonía. El uso masivo de cocinas mejoradas fue considerado como una solución a la contaminación intradomicilaria; sin embargo, las evidencias revelan que muchas de las cocinas mejoradas no logran reducir los niveles de contaminantes a valores permisibles ${ }^{(6)}$. Esto sugiere la necesidad de evaluar el impacto en salud en el Perú de las distintas marcas de cocinas mejoradas implementadas.

En relación al agua, se hizo hincapié en la necesidad de dotar agua segura, servicios de eliminación de excretas y mejorar hábitos de higiene para mejorar la salud. Se evidenció la falta de investigación en Perú del impacto en la salud de la contaminación de agua con arsénico. En relación al cambio climático se ha observado en 